\title{
Philosophiques
}

\section{Explication et justification en philosophie morale}

\section{Jocelyne Couture}

Volume 28, numéro 1, printemps 2001

La nature des normes

URI : https://id.erudit.org/iderudit/004912ar

DOI : https://doi.org/10.7202/004912ar

Aller au sommaire du numéro

Éditeur(s)

Société de philosophie du Québec

ISSN

0316-2923 (imprimé)

1492-1391 (numérique)

Découvrir la revue

Citer cet article

Couture, J. (2001). Explication et justification en philosophie morale.

Philosophiques, 28(1), 129-150. https://doi.org/10.7202/004912ar

\section{Résumé de l'article}

L'hypothèse qui guide le présent article est que les méthodes et techniques de dérivation ou de modélisation utilisées en éthique contemporaine, ont une incidence sur les contenus proprement moraux de la théorie morale ; vues comme de simples outils à l'usage du philosophe moral, elles agissent en fait comme des sources de normativité morale. Cette hypothèse n'est pas une simple reformulation de l'idée voulant qu'il existe, au sein de la théorie morale, un rapport entre explication et justification ou entre méta-éthique et éthique. Pour le montrer, je m'appuierai d'abord sur une caractéristisation générale de ces composantes de la théorie morale et des rapports qu'elles entretiennent entre elles. Dans un deuxième temps j'arguerai que l'usage d'outils méthodologiques particuliers peut avoir une incidence sur les contenus moraux proprement dits. Pour caractériser cette incidence et en décrire les mécanismes, je ferai d'abord appel à une approche pragmatique de l'explication, puis je donnerai deux exemples, tirés de la philosophie morale contemporaine, et illustrant le fait que certains contenus moraux ainsi introduits échappent de fait à une justification en bonne et due forme.
Ce document est protégé par la loi sur le droit d'auteur. L'utilisation des services d'Érudit (y compris la reproduction) est assujettie à sa politique d'utilisation que vous pouvez consulter en ligne.

https://apropos.erudit.org/fr/usagers/politique-dutilisation/ 


\title{
Explication et justification en philosophie morale
}

JOCELYNE COUTURE

Université du Québec à Montréal

couture.j@uqam.ca

RÉSUMÉ. - L'hypothèse qui guide le présent article est que les méthodes et techniques de dérivation ou de modélisation utilisées en éthique contemporaine, ont une incidence sur les contenus proprement moraux de la théorie morale; vues comme de simples outils à l'usage du philosophe moral, elles agissent en fait comme des sources de normativité morale. Cette hypothèse n'est pas une simple reformulation de l'idée voulant qu'il existe, au sein de la théorie morale, un rapport entre explication et justification ou entre méta-éthique et éthique. Pour le montrer, je m'appuierai d'abord sur une caractéristisation générale de ces composantes de la théorie morale et des rapports qu'elles entretiennent entre elles. Dans un deuxième temps j'arguerai que l'usage d'outils méthodologiques particuliers peut avoir une incidence sur les contenus moraux proprement dits. Pour caractériser cette incidence et en décrire les mécanismes, je ferai d'abord appel à une approche pragmatique de l'explication, puis je donnerai deux exemples, tirés de la philosophie morale contemporaine, et illustrant le fait que certains contenus moraux ainsi introduits échappent de fait à une justification en bonne et due forme.

\begin{abstract}
The central claim made in this article is that the various methodological devices used by contemporary moral philosophers in order to represent or derive moral principles affect the very moral content of their theory; seen by them as mere tools for philosophers, they in fact are sources of moral normativity. This claim is not a mere reformulation of the idea that there is within moral theory a connection between explanation and justification or between metaethics and ethics. To show that this is so, I first give a general characterization of the various components of moral theory and of their relationships. I then argue that the use of particular methodological devices affect the very moral content of the theory. To characterize such incidence and its mechanisms, I first appeal to a pragmatic conception of explanation and, finally, I discuss two examples from contemporary moral theory showing that substantive moral claims so introduced, in fact fail to be justified.
\end{abstract}

\section{Introduction}

Dans le présent contexte philosophique l'idée d'une réflexion d'ensemble sur les méthodes et sur les techniques de représentation, de modélisation ou de dérivation utilisées en éthique contemporaine, ne semble guère soulever d'enthousiasme. En plus de ressusciter le spectre d'une méta-éthique qui, durant la première moitié du vingtième siècle, aurait contribué, au dire de certains, à la sclérose de la philosophie morale, le projet d'une telle réflexion se heurte à la croyance, que semblent entretenir plusieurs philosophes, selon laquelle des tâches plus urgentes attendent la philosophie morale, comme 
celles d'articuler et de défendre une conception de la justification morale et, bien sûr, de justifier conformément des jugements moraux particuliers. Mais c'est bien vite passer sous silence le fait que, dans plusieurs théories contemporaines, la justification des jugements moraux n'est pas redevable qu'à des conceptions philosophiques concernant le sens, la portée ou les limites de la justification morale, la nature des normes ou encore la nature des raisons susceptibles d'accréditer un jugement moral. La justification des jugements moraux particuliers est aussi redevable aux modèles théoriques, souvent issus des diverses disciplines des sciences sociales, et utilisés à titre de méthodes de dérivation de ces jugements.

L'analyse de ces modèles et du rôle qu'ils jouent dans la détermination du contenu des jugements moraux est à mon avis une tâche urgente de la philosophie morale. En effet, l'hypothèse qui guide le présent article est que ces méthodes et techniques de dérivation ou de modélisation ont une incidence, et une incidence souvent méconnue par ceux-là mêmes qui en font usage, sur les contenus proprement moraux de la théorie morale ; vues comme de simples techniques à l'usage du philosophe moral, elles sont en fait des sources de normativité morale. Si cette hypothèse a quelque plausibilité, et c'est ce que j'essayerai de montrer ici, alors il importe, pour la philosophie morale d'apprendre à distinguer ce qui, dans les normes morales avancées par les philosophes, découle de leur conception de la justification morale et ce qui relève au contraire de l'appareil méthodologique dont il font usage ; ce qui est justifié aux termes de la théorie morale et ce qui ne l'est pas. Et c'est aussi une tâche urgente que de mettre au point une approche systématique de l'éthique qui puisse nous permettre de comprendre exactement le rôle que peuvent jouer ces diverses composantes méthodologiques dans la conceptualisation et l'articulation des normes morales.

Il ne faudrait pas s'attendre à ce que ces tâches monumentales soient effectuées dans le cours du présent article. J'aimerais toutefois montrer ici qu'il existe de bonnes raisons d'élargir un peu le cadre de la philosophie morale de façon à pouvoir y inclure une réflexion critique sur sa propre pratique théorique et, en particulier, sur la portée d'une justification philosophique des jugements moraux. Dans cet article, j'ai d'abord voulu montrer comment les différentes composantes de la théorie morale contribuent respectivement à la détermination des contenus moraux proprement dits. Pour ce faire, il m'est apparu profitable d'adopter la perspective des rapports qu'entretiennent l'explication et la justification au sein de la théorie morale ; ces rapports, selon moi, existent à deux niveaux différents : celui ou s'élabore une conception ou une théorie de la justification morale et celui de la justification proprement dite des jugements moraux. C'est à ce second niveau que la justification fait le plus souvent intervenir, sous forme des techniques particulières de représentation, de modélisation ou de dérivation, des schémas explicatifs qui ont une incidence directe sur les contenus moraux. Pour caractériser cette incidence, et en décrire les mécanismes, je fais appel à 
une approche pragmatique de l'explication. Pour terminer, j'illustre par deux exemples, la manière dont cette approche s'applique à la théorie morale tout en montrant que certains contenus moraux, que les philosophes estiment justifiés, échappent de fait à une justification en bonne et due forme.

\section{Les objectifs de la théorie morale}

Selon une conception assez répandue, mais à mon avis inadéquate, de la philosophie morale, celle-ci se caractérise par la poursuite de deux objectifs théoriques. Thomas Scanlon ${ }^{1}$, par exemple, y distingue ce qu'il appelle "l'enquête philosophique » et "l'enquête morale ». L'enquête morale, dit-il, est « une enquête sur la recevabilité des jugements moraux particuliers ainsi que des raisons morales que nous faisons valoir en faveur ou à l'encontre de tels jugements. " ${ }^{2}$. L'objectif de l'enquête morale serait donc de justifier certains principes moraux en établissant la supériorité, d'un point de vue moral, des raisons particulières qui militent en faveur de ces principes. L'enquête philosophique, pour sa part, est selon Scanlon, " une enquête sur la nature de la moralité [qui] cherche à répondre à des questions telles que les suivantes : A quel genre de questions essayons-nous de répondre lorsque nous tentons de réfléchir à ce qui est bien ou à ce qui est mal ? Quel genre de faits, ou de vérité, si cela existe, peuvent correspondre aux réponses que nous donnons à ces questions ? Comment pouvons-nous découvrir des vérités de ce type ? Pourquoi ont-elles tellement d'importance pour nous ? C'est-à-dire, à quel genre de raison d'agir correspondent-elles et comment ces raisons peuvent elles avoir le type de priorité, qu'on attribue communément à la moralité, sur d'autres considérations ? " ${ }^{3}$. L'objectif de l'enquête philosophique serait donc de proposer une explication de la moralité ou de certains de ses aspects ${ }^{4}$.

Ainsi conçues, l'enquête philosophique et l'enquête morale sont-elles reliées l'une à l'autre ? Toute une tradition métaéthique nous a enseigné qu'elles ne le sont pas; que ce que nous disons ou croyons du savoir moral, du raisonnement moral, de la signification des concepts moraux ou de la

1. Scanlon, 1992

2. Ibid., p. 4

3. Ibid., p. 5

4. Le terme "explication " doit ici être pris dans le sens large que lui confèrent les sciences sociales. Pour n'en donner que quelques exemples, les explications proposées dans le cadre de l'enquête philosophique sont tantôt logiques, lorsqu'il s'agit par exemple de rendre compte de la structure des raisonnements moraux ; elles sont tantôt épistémologiques, lorsqu'elles cherchent à rendre compte de la nature ou de la possibilité d'un savoir moral ; elles peuvent faire appel à une philosophie de l'action, lorsqu'elles cherchent à caractériser les rapports entre croyances et actions; elles sont tantôt sociologiques lorsqu'elles cherchent à rendre compte de la fonction ou du développement des pratiques morales et elles prennent quelquefois la forme de fables philosophiques au sujet d'un hypothétique état de nature, lorsqu'elles cherchent à rendre compte d'une morale par consensus. 
structure du raisonnement moral, n'a rien à voir avec les jugements moraux substantiels que nous sommes prêts à endosser ou à rejeter ${ }^{5}$. Bref, les philosophes qui appartiennent à cette tradition et veulent la perpétuer, croient que les explications données dans le cadre de l'enquête philosophique sont moralement neutres et laissent entièrement ouvert le contenu de la morale. Il n'est pas difficile de voir que cette croyance est erronée. Si quelqu'un adhère à une conception conséquentialiste du raisonnement moral, il ne peut pas, du moins d'une façon cohérente, défendre ensuite un principe moral en vertu duquel on devrait toujours dire la vérité ; car, en certaines circonstances, dire la vérité, a des conséquences indésirables du point de vue moral. Si quelqu'un défend une conception de la moralité en vertu de laquelle toutes les obligations morales sont relatives à des contextes culturels et sociaux particuliers, il ne peut pas ensuite soutenir que les relations entre les peuples devraient être régies par une certaine conception des droits de la personne. Si quelqu'un croit qu'un principe moral est un principe auquel il est rationnel pour tous de se conformer, il ne peut pas ensuite défendre un principe de justice en vertu duquel la répartition des avantages sociaux devrait être fonction de l'hérédité. Cette personne ne pourrait pas non plus défendre, en tant que conception substantielle de la justice, une doctrine qui laisserait entièrement indéterminées des questions comme celle de la répartition sociale des devoirs et des privilèges, car une telle doctrine exigerait des agents, que l'on a supposé rationnels, qu'ils s'exposent délibérément à l'arbitraire de circonstances ou de décisions qui pourraient leur être préjudiciables.

Comment donc caractériser de telles relations entre l'enquête philosophique et l'enquête morale ? Si l'on s'en remet à la dichotomie établie par Scanlon, ce qu'il s'agit de caractériser est le rapport entre une explication de la moralité et la justification morale ; ainsi, les exemples précédents ne feraient que montrer la consistance à laquelle on doit s'attendre, entre une caractérisation générale de ce que c'est que d'être bien ou juste et une spécification des choses ou des actions particulières que nous jugeons bonnes ou justes. Mais comment peut-on adopter cette façon de voir les choses sans gommer une différence importante, dont on devrait pouvoir rendre compte, entre l'explication et la justification morale ? Tandis que la première cherche à rendre compte de ce qui est, (au sujet de la moralité, du raisonnement moral, de la vérité, des croyances et ainsi de suite), la seconde vise à établir ce qui devrait être d'un point de vue moral. Prétendre dans ces circonstances que le rapport entre le général et le particulier est au coeur, ou constitue

5. Les positions d'un philosophe comme J. L. Mackie sont tout à fait représentatives de cette tradition. " These are first-order moral view, positive or negative : the person who adopts either of them is taking a certain practical, normative, stand. By contrast, what I am discussing is a second-order view, a view about the status of moral values and the nature of moral valuing, about where and how they fit the world. The first- and second-order views are not merely distinct but completely independent : one could be a second-order sceptic without being a firstorder one, or again, the other way round ». Mackie, 1977, p. 16. 
l'essentiel, des relations qui existent entre l'enquête philosophique et l'enquête morale nous forcerait à admettre que les explications proposées dans le cadre de la première véhiculent déjà un contenu moral normatif et qu'elles le véhiculent de façon telle que la théorie morale serait irrémédiablement vouée à la circularité. A la question, qui relève de la justification morale : "Pourquoi cette action particulière est-elle moralement bonne ? ", on devrait répondre, mais en faisant appel à une explication de la moralité : «Parce que cette action particulière a toutes les propriétés auxquelles nous nous référons lorsque nous disons qu'une action est bonne ». Et à la question, qui relève d'une explication de la moralité : "Pourquoi devrions nous vouloir dire telle et telle chose lorsque nous disons qu'une action est bonne ? ", on devrait répondre, en faisant appel à une justification morale : "Ces choses particulières que nous estimons bonnes ont les propriétés auxquelles nous nous référons lorsque nous disons qu'une chose est bonne ». Concevoir la relation entre l'enquête philosophique et de l'enquête morale comme un rapport direct entre l'explication de la moralité et la justification morale nous conduit donc à une impasse : cette façon de voir les choses ne permet de rendre compte, ni de la différence qui existe entre les visées respectives de ces deux enquêtes, ni de l'hétérogénéité de leurs objets respectifs, ni du rôle que jouent les explications dans la théorie morale.

Il me semble que la réponse que nous devrions pouvoir donner, en théorie morale, lorsque nous demandons « Pourquoi devrions-nous croire que cette chose particulière est juste ? " n'est pas, " Parce qu'elle a toutes les propriétés auxquelles nous nous référons lorsque nous disons que quelque chose est moralement juste ", mais plutôt «Parce que nous avons des raisons non morales de croire que les raisons morales que nous avançons pour soutenir que cette chose est juste sont elles-mêmes de bonnes raisons ». Ou dans une formulation qui évite toute ambiguïté au sujet des «bonnes raisons": "Parce que nous disposons d'une explication de la moralité (du raisonnement moral, de la motivation morale) qui nous permet de voir que les raisons morales que nous avançons pour soutenir qu'une chose est juste, sont des raisons valides. Et si nous demandons : « Pourquoi devrions nous souscrire à une telle explication de la moralité ? " la réponse ne nous ramène pas directement aux " choses particulières que nous estimons être moralement justes » mais bien plutôt au domaine indépendant des explications rivales de la moralité. Si un conséquentialiste ne peut pas soutenir que nous devons dire la vérité en toutes circonstances, ce n'est pas, à strictement parler, parce que dire la vérité a parfois des conséquences non désirables du point de vue moral, mais parce que les raisons qu'il pourrait avancer pour soutenir que nous devons toujours dire la vérité ne sont pas valides en regard des standards conséquentialistes.

De ce point de vue, il apparaît que les explications données dans le cadre de l'enquête philosophique contribuent à l'élaboration d'une conception de la justification et qu'à ce titre, elles contribuent à déterminer les stan- 


\section{Philosophiques / Printemps 2001}

dards et les limites de la justification morale proprement dite. L'enquête philosophique a donc finalement une visée normative, bien que la normativité qui la caractérise ne soit pas de nature morale. Cette façon de voir la théorie morale rend compte de la relation qui existe entre l'enquête philosophique et l'enquête morale sans confondre les deux. Elle rend compte aussi des contraintes que la première fait peser sur le contenu de la morale. Ces contraintes ne sont pas elles-mêmes morales, elles ne sont pas non plus sémantiques, elles sont plutôt méthodologiques. L'enquête philosophique, comme le dit Norman Daniels dans d'autres termes, fournit à la fois « une conception théorique de ce qu'est la justification en éthique et un processus adapté à la résolution de problèmes moraux ${ }^{6} »$. Si, comme je le crois, Daniels a raison sur ce point, alors, l'enquête morale et l'enquête philosophique, telles que conçues par Scanlon, doivent être revisitées.

La bipartition qu'établit Scanlon entre les deux objectifs théoriques de la philosophie morale suggère une nette bipartition, au sein de celle-ci, entre l'explication, qui serait la tâche de l'enquête philosophique, et la justification qui reviendrait à l'enquête morale. Or, si l'on peut d'emblée convenir que l'enquête philosophique chercher à articuler des explications de la moralité ou de certains de ses aspects, il faut bien voir aussi que c'est dans un but normatif qu'elle le fait, à savoir, dans le but de déterminer les standards de la justification morale. Et si l'on convient d'emblée que l'enquête morale a pour objectif la justification des jugements moraux particuliers il faut bien voir aussi que la validité d'une telle justification dépend de sa capacité de se conformer à la conception de la justification issue de l'enquête philosophique. De plus, Scanlon pense que l'enquête morale consiste à donner des raisons morales à l'appui des jugements moraux particuliers. D'une part, il semble ainsi exclure a priori la possibilité que l'enquête morale fasse intervenir, outre des raisons morales, des raisons qui relèveraient, par exemple, de notre compréhension de la société, de son fonctionnement ou des mécanismes qui régissent les interactions sociales au sein de ses différents secteurs. Il semble ainsi exclure a priori, et à tort, j'en donnerai bientôt un exemple, que l'enquête morale fasse intervenir, elle aussi, des explications, et du même coup, que des explications autres que des explications de la moralité puissent trouver leur place au sein de la théorie morale. D'autre part, si, comme je le crois, le but de l'enquête philosophique est de fixer les standards de la justification, alors il faut bien admettre la possibilité, j'en donnerai un exemple plus bas, qu'en vertu de certaines enquêtes philosophiques, aucune raison proprement morale ne soit requise pour la justification des principes moraux particuliers. Mais même dans les cas, sans doute plus fréquents, ou des raisons morales sont requises pour qu'un principe soit justifié moralement, il est clair que la justification ne peut pas reposer entièrement sur des raisons de cette nature. Si, en effet, les explications données dans le cours de l'enquête

6. Daniels, 1996, p.1. 
philosophique contribuent à fixer les standards de la justification, elles doivent aussi constituer, le cas échéant avec les raisons morales, les prémisses de tout raisonnement dont la conclusion est un jugement moral particulier. Pour le dire en d'autres mots, la structure logique de la justification des jugements moraux particuliers devrait montrer que la justification fait intervenir, conjointement ou non avec des raisons morales, des raisons non morales qui relèvent d'une enquête philosophique sur la nature de la moralité.

Je donnerai rapidement deux exemples pour montrer comment la pratique théorique des philosophes moraux contemporains endosse effectivement cette conception du rapport entre l'enquête philosophique et l'enquête morale. Mon premier exemple est celui des théories, maintenant de plus en plus nombreuses, qui font appel à ce qu'il est convenu d'appeler l'équilibre réfléchi ${ }^{7}$. L'équilibre réfléchi est d'abord un modèle - une explication - de la manière dont nous formons et abandonnons des croyances; n'importe quelle croyance, aux dires de ses défenseurs. L'idée centrale en est que nous testons les différents éléments d'un système de croyances - supposons un système de croyances morales - en cherchant à les rendre cohérents entre eux, c'est-à-dire en cherchant des principes plus généraux ou plus abstraits susceptibles d'exprimer ce que ces croyances ont en commun. L'exercice suppose un processus d'ajustement mutuel — une mise en équilibre - des principes et des croyances au terme duquel certaines croyances devront être abandonnées ou, tout au moins, révisées. Lorsque John Rawls introduit ce modèle dans $A$ Theory of Justice, il le décrit d'abord comme étant le mode de raisonnement approprié, entre autres choses, en matières de morale. Il l'utilise ensuite comme procédé de justification de ses propres principes de justice. Partant de ce qu'il appelle nos jugements moraux bien pesés, ces jugements particuliers qu'après mûre réflexion nous refuserions de renier, il montre qu'il peuvent être subsumés sous deux principes de justice qu'il estime dès lors justifiés moralement. Son explication du raisonnement moral est, dans les termes que j'ai utilisés plus tôt, une théorie de la justification fixant le domaine et la nature des raisons morales qui peuvent légitimement intervenir dans la justification morale proprement dite des principes moraux particuliers.

Mon deuxième exemple illustre le cas où la justification de jugements moraux particuliers, sans faire appel à des raisons de nature morale, évite néanmoins de se confondre avec «l'enquête philosophique sur la nature de la moralité ». Cet exemple montre aussi comment des explications peuvent intervenir directement dans la justification des jugements moraux particuliers, et non seulement dans l'enquête philosophique. Dans Morals by Agreement ${ }^{8}$,

7. Norman Daniels, Kai Nielsen et plusieurs autres établissent une distinction entre l'équilibre réfléchi large et l'équilibre réfléchi étroit. Cette distinction n'affecte en rien la caractérisation générale que je donne ici de l'équilibre réfléchi. Daniels, 1996, 21-46, Nielsen, 1996, 13-21, 219-223.

8. Gauthier, 1986 
David Gauthier emprunte à la théorie de la négociation rationnelle un modèle du choix rationnel. Selon la dichotomie établie par Scanlon, il aurait fallu s'attendre à ce que ce modèle, puisqu'il est un modèle explicatif, fasse partie de l'enquête philosophique. Mais si cette dernière constitue, comme je le crois, une conception de la justification alors il aurait de surcroît fallu s'attendre à ce que Gauthier, après avoir introduit ce modèle comme une explication du raisonnement pratique tel qu'il intervient dans le domaine de la moralité, l'utilise ensuite pour montrer qu'en suivant les règles du choix rationnel, en matière de morale, nous arrivons à sélectionner certains principes de justice plutôt que d'autres. Mais ce n'est pas ce que fait Gauthier ; il propose plutôt comme principe de justice, un principe ( le principe de la concession relative minimax) qui, dans son modèle, sert à faire des choix rationnels c'est-à-dire, un principe qui ne doit apparemment sa validité qu'à la conception du choix rationnel dont il est issu. En vertu de la distinction de Scanlon, ce principe ne serait pas justifié car il ne fait appel à aucune raison morale. En outre il faudrait conclure que l'enquête philosophique, puisqu'elle est la seule, selon Scanlon à proposer des explications, s'est entièrement, et d'une façon illégitime, substituée à la justification morale proprement dite. Je ne crois pas qu'une telle conclusion s'impose. La thèse centrale de Morals by Agreement est que la moralité, à toutes fins pratiques, n'est rien d'autre que l'exercice de la rationalité instrumentale. Cette thèse ne peut être soutenue qu'en alléguant, comme le fait Gauthier, d'une explication de la moralité en vertu de laquelle aucune prémisse morale n'est requise pour la justification de principes de justice. C'est en pure conformité avec cette conception de la justification morale, que Gauthier fait ensuite appel à un modèle explicatif du choix rationnel et estime justifié, en tant que principe de justice substantielle, un principe qui ne fait appel à aucune prémisses morales. S'il apparaît à première vue que l'enquête philosophique chez Gauthier, se confond avec la justification de jugements moraux particuliers, ou encore s'y substitue, c'est que l'on confond dès le départ une conception de la justification et la justification proprement dite d'un principe donné ainsi que le rôle que l'une et l'autre sont appelées à jouer au sein de la théorie morale.

\section{Explications et présuppositions : une approche pragmatique}

Le rôle que jouent les explications formulées dans le cours de l'enquête philosophique n'accrédite nullement, comme nous venons de le voir, l'idée selon laquelle une explication de la moralité devrait être neutre par rapport aux contenus moraux proprement dits. Puisqu'une théorie de la moralité est aussi une théorie de la justification, elle impose effectivement des contraintes sur le domaine des raisons qui peuvent être alléguées en faveur de jugements moraux particuliers et, indirectement, sur la nature des jugements susceptibles d'être justifiés. Il faut cependant garder présent à l'esprit que l'absence de neutralité, ainsi entendue, ne se manifeste que sous la forme de contraintes sur le type des arguments ou des explications qui peuvent être utilisés dans 
le contexte de la justification morale. Loin de constituer un empiètement direct sur les contenus moraux de la théorie morale, ces contraintes se portent au contraire garantes de la cohérence et de la validité internes de celle-ci.

Hors de ces limites, c'est avec raison que l'on peut s'inquiéter de l'absence de neutralité des explications données dans le cadre de l'enquête philosophique. Certaines de ces explications en effet ne sont pas neutres, au sens où elles réussissent à imposer des contraintes substantielles - et non pas seulement formelles - sur le contenu des principes moraux. L'absence de neutralité dans ce sens est celle que l'on s'attend à trouver lorsque les explications de la moralité véhiculent, d'une manière ou de l'autre, des présuppositions qui affectent les contenus moraux de la théorie. La faiblesse des théories morales où l'on trouve de telles explications de la moralité, réside en ce qu'elles soustraient à la justification proprement dite, cette partie de leur contenu moral qui dépend directement des présupposés de l'explication.

Une façon triviale pour une explication d'être non-neutre dans ce sens et avec ce résultat, est de présupposer une évaluation morale. Lorsque Hobbes, par exemple, explique l'émergence de la moralité en montrant qu'elle constitue la seule issue possible pour des personnes égales en force et en intelligence et uniquement guidées par la poursuite de leurs intérêts individuels, il est clair que son explication contient déjà, sous la forme d'une conception de la personne, une évaluation morale de ses semblables. On peut très bien comprendre comment cette conception de la personne, et le présupposé moral qu'elle véhicule, débouchent sur une doctrine politique autoritaire mais ni l'autoritarisme en tant que contenu normatif, ni l'évaluation morale qui lui sert de prémisse, ne sont justifiés de façon indépendante.

Certaines explications aboutissent à des résultats similaires du point de vue de la justification parce qu'elles véhiculent des présuppositions, non pas morales, mais structurales. Les présuppositions de ce type sont plus difficiles à détecter mais, comme nous le verrons dans la suite de cet article, certaines d'entre elles du moins, peuvent être mises en évidence grâce une analyse pragmatique de l'explication. Je présenterai brièvement les lignes directrices d'une telle analyse avant de montrer comment elle peut s'appliquer à la théorie morale.

L'idée d'une pragmatique de l'explication n'est pas nouvelle, mais elle a été articulée d'une façon particulièrement intéressante par Alan Garfinkel, dans un ouvrage qui, à ma connaissance, n'a jamais reçu toute l'attention qu'il méritait ${ }^{9}$. Dans la perspective spécifique mise de l'avant par une pragmatique de l'explication, toute explication est relative à un contexte. L'une des premières difficultés que doit surmonter cette approche est par conséquent de parvenir à une caractérisation suffisamment précise de ce qui constitue le contexte d'une explication. Selon Garfinkel, il serait vain d'en chercher une caractérisation syntaxique ; il propose plutôt de l'analyser en terme d'espace contrasté (contrast space). L'idée intuitive qui guide son analyse est que l'explication, en

9. Garfinkel, 1981 


\section{Philosophiques / Printemps 2001}

tant que réponse à une question préthéorique et habituellement ambiguë, sélectionne une interprétation possible de cette question et crée, par conséquent, son propre espace, différent de celui que créerait une autre explication répondant, cette fois-ci, à la question interprétée différemment. Ainsi l'espace contrasté d'une explication - ce qu'elle cherche à expliquer par opposition à ce qu'elle ne cherche pas à expliquer - constitue le contexte que l'on doit prendre en considération pour juger de sa pertinence ou de son bien-fondé. Pour illustrer son propos, Garfinkel emprunte à Fred Dreske ${ }^{10}$ l'exemple suivant : Supposons que X vient de prêter 300 \$ à Y et que Z, témoin de la scène, demande à $\mathrm{X}$ : " Pourquoi as-tu prêté 300 \$ à Y ? ». La réponse de $\mathrm{X}$ est qu'il a prêté cette somme à Y parce que c'est la somme dont Y avait besoin. Mais $\mathrm{X}$ aurait pu répondre différemment à la question de $\mathrm{Z}$ : il aurait pu dire, par exemple, qu'il a prêté 300 \$à Y parce que le père de Y n'était pas là pour la lui prêter. Ou encore, qu'il a prêté cette somme à Y parce que c'est Y et non pas V qui la lui a demandée. Ou bien qu'il a prêté cette somme à Y parce qu'il ne voulait pas la lui donner. En répondant comme il l'a fait, $\mathrm{X}$ a sélectionné l'un des sens possible de la question de Z, à savoir, "pourquoi as-tu prêté 300 \$ à Y plutôt que 500 \$ ou 200 \$ ? » Dans les termes de Garfinkel, X a ainsi crée l'espace propre de son explication, distinct de celui des autres explications qu'il aurait pu donner et qui constitue le contexte dans laquelle sa réponse est pertinente.

Souvent, dit Garfinkel, ce que nous considérons comme des explications rivales n'en sont pas du tout, puisqu'elles sélectionnent en fait des espaces différents, c'est-à-dire qu'elles répondent à des questions différentes. Ainsi, dit-il, la physique médiévale et celle de Newton ne sont pas des explications rivales du mouvement, parce qu'elles ne fonctionnent pas dans le même espace contrasté : alors que les physiciens du Moyen-Age expliquaient pourquoi une chose donnée se meut au temps $\mathrm{t}$ - plutôt que d'être immobile - Newton expliquait pourquoi une chose donnée possède une accélération donnée au temps $\mathrm{t}$ - plutôt que telle autre accélération ${ }^{11}$. Cette analyse permet aussi de caractériser plus précisément ce qu'il convient d'entendre par une bonne, ou une mauvaise, explication. Une bonne explication, dit Garfinkel, est une explication qui a le même espace contrasté que la question à laquelle elle veut répondre. La plupart du temps, dit-il, les explications ne répondent pas aux questions préthéoriques que nous avons en tête et, en conséquence, nous ne savons pas, la plupart du temps, ce qu'elles expliquent.

Le fait que les explications soient relatives à un contexte donné, caractérisé à la manière de Garfinkel, permet donc de comprendre les mécanismes grâce auxquels les explications peuvent véhiculer, souvent à l'insu de ceux qui les formulent, des présuppositions concernant, par exemple, l'importance de certaines questions par rapport à d'autres, l'orientation de nos intérêts cogni-

10. L'exemple est tiré de (Dretske 1973, p. 419) et il est présenté dans Garfinkel, 1991, p. 24

11. Garfinkel, 1991, p. 8, 25-26 
tifs, voire la forme d'une représentation adéquate du monde. Sur ce dernier point en particulier, il importe de prêter une attention spéciale, selon Garfinkel, à une catégorie de présuppositions qu'il appelle les présuppositions structurales. Dans les sciences sociales, dit-il, les explications impliquant des individus qui sont eux-mêmes reliés à des systèmes, sont souvent le véhicule de ce type de présuppositions. Ici encore, Garfinkel illustre son propos par des exemples ${ }^{12}$. Supposons, dit-il, qu'un professeur ait décidé d'attribuer leur note à ses étudiants selon une courbe de distribution en vertu de laquelle seul le meilleur essai doit recevoir la mention A. Il s'avère que le meilleur essai de la classe est celui de Thérèse. Un collègue de ce professeur, s'enquérant auprès de celui-ci de la raison pour laquelle Thérèse a obtenu la mention $\mathrm{A}$, reçoit cette réponse : "Thérèse a remis un essai rigoureux, bien documenté, bien écrit, et qui témoigne d'une réelle connaissance de son sujet ». Mais si le collègue insiste et demande encore si tous les étudiants qui ont produit un travail rigoureux, bien documenté, bien écrit et qui témoigne d'une réelle connaissance de leur sujet ont obtenu un A, la réponse ne peut être que négative puisque qu'il était décidé à l'avance qu'il n'y aurait qu'un essai, le meilleur de la classe, qui recevrait la mention $\mathrm{A}$. Mais puisque tel est le cas, la réponse à la première question constitue une explication partielle du fait que Thérèse ait obtenu la mention A. C'est une explication qui ne tient compte que des faits concernant Thérèse et qui laisse dans l'ombre le fait relatif qu'elle ait écrit le meilleur travail de la classe, de même que cet autre fait relatif que seul le meilleur travail devait recevoir un A. Ces deux faits, relatifs à la structure ou au système dans lequel Thérèse est placée, sont présupposés par la première explication, dans le sens où celle-ci, plutôt que de répondre à la question posée, répond plutôt à cette autre question : «Etant donné qu'il était décidé à l'avance que seul le meilleur essai de la classe se mériterait la mention $\mathrm{A}$, pourquoi Thérèse a-t-elle reçu la mention $A$ ? ». C'est ce type de présupposition que Garfinkel nomme présupposition structurale et, selon lui, le test qui révèle la présence de telles présuppositions dans des explications est, comme dans le présent exemple, l'impossibilité de généraliser l'explication.

Selon Garfinkel, les défenseurs de l'individualisme méthodologique ou encore du réductionnisme n'ont pas suffisamment réalisé les conséquences, pour une théorie de l'explication, des principes épistémologiques qu'ils défendaient : les explications faisant intervenir des systèmes ou des macrophénomènes n'ont pas, dit il, le même espace de contraste que les explications qui ne font intervenir que des individus, des propriétés individuelles ou des microphénomènes. Par conséquent, elles n'expliquent pas les mêmes choses ${ }^{13}$. C'est d'abord faire preuve d'un apriorisme considérable au sujet de ce qu'est une représentation adéquate de l'univers, que de croire que les seules explications acceptables d'un point de vue épistémologique et les seules qui soient

12. Ibid., p. 41-45

13. Ibid., p. 14-19 
susceptibles de faire progresser les connaissances soient des explications du second type. De plus, les explications du deuxième type contiennent, plus souvent qu'autrement, des présuppositions structurales, c'est-à-dire qu'elles négligent de prendre en compte les effets structuraux des systèmes plus vastes auxquels les individus sont liés et qui sont, de fait, des composantes inéliminables de ce que l'on cherche à expliquer.

\section{Explication et justification morale}

J'ai souligné à quelques reprises que les explications en théorie morale, n'interviennent pas uniquement dans le cadre de l'enquête philosophique mais aussi dans le cadre de l'enquête morale. Tel est, par exemple, le cas de la théorie de la négociation rationnelle que David Gauthier utilise comme modèle de la rationalité instrumentale et qui, comme nous l'avons vu, satisfait aux exigences de la justification posées par son explication de la moralité. C'est aussi le cas des explications de nature économique que l'on trouve dans certaines théories de la justice, ou d'explications de nature sociologique ou anthropologique qui viennent étayer l'argumentation en faveur de principes moraux substantiels. C'est au sujet de telles explications, qui ne sont pas des explications de la moralité, mais qui contribuent à la justification proprement dite, que je veux maintenant montrer qu'elles véhiculent parfois des présuppositions structurales qui affectent de façon subreptice les contenus proprement moraux de la théorie. J'en donnerai maintenant deux exemples avant de revenir ensuite aux explications de la moralité susceptibles de contenir, elles aussi, des présuppositions structurales.

1- Mon premier exemple est celui de la justice économique. Cette partie de la justice sociale cherche à articuler les principes et les mécanismes d'une distribution équitable de la richesse. Préalablement à toute argumentation morale portant sur des principes de justice particuliers, il est spécialement important pour les théories de ce type, de faire appel à des théories explicatives susceptibles de rendre compte de plusieurs phénomènes économiques empiriques reliés à la circulation, à l'acquisition, au transfert et à la production de la richesse et parfois à des modèles de l'agent économique. Une fois ces questions clarifiées, il s'agit ensuite d'évaluer, d'un point de vue moral, le bien fondé des mécanismes qui président actuellement à la distribution de la richesse, de formuler, le cas échéant, des règles ou des principes moraux susceptibles de les rendre plus équitables et, de déterminer, d'un point de vue empirique, la faisabilité des mécanismes qui peuvent contribuer à l'application de ces principes.

Certaines explications de la distribution de la richesse dans les sociétés actuelles mettent l'accent sur les caractéristiques individuelles des agents : leurs efforts, leurs talents ou leur capacité d'adaptation seraient, selon ces explications, les facteurs déterminants de l'existence des disparités économiques entre les individus. Supposons pour simplifier que cette explication soit 
tenu pour supérieure à toute autre et qu'elle constitue la seule base à partir de laquelle on procède à l'évaluation morale des mécanismes de la distribution de la richesse. Sur la base d'une telle explication, on peut estimer que les mécanismes actuels de la distribution économique sont injustes et qu'ils doivent être rectifiés et on peut aussi estimer le contraire. Par exemple, on peut articuler et défendre un principe de justice visant à rectifier la distribution initiale, et en vertu duquel l'on devrait supporter économiquement les personnes moins talentueuses ou plus faibles. Ou au contraire, on peut arguer que la distribution initiale est naturelle et que d'un point de vue moral il ne faut ni la modifier, ni même songer à en déjouer les mécanismes. On peut même ajouter, dans cet esprit, que la plus grande richesse récompense les plus méritants, et qu'ils en seraient injustement privés si l'on changeait les règles du jeu ou si on procédait à une redistribution des richesses. Mais que l'on argue pour une amélioration des mécanismes actuels de la distribution des richesses ou pour le statu quo en cette matière, les arguments ne porteront toujours que sur les facteurs de la distribution identifiés par l'explication initiale, à savoir, les efforts, les talents ou les capacités d'adaptation des individus et jamais sur d'autres facteurs possibles.

Supposons au contraire une théorie de la justice économique qui se donnerait comme seul point de départ une explication systémique de la distribution des richesses. Une telle explication met l'accent non pas sur les caractéristiques individuelles des agents, bien qu'elle en tienne aussi compte, mais plutôt sur la place respective qu'ils occupent au sein d'une distribution donnée. Les disparités dans la richesses sont ainsi attribuées au fait que les personnes font partie d'un même système socio-économique au sein duquel la prospérité des uns s'acquiert aux dépens de celle des autres. Dans cette perspective, la propriété matérielle n'est plus vue comme une relation entre une personne particulière et les choses qu'elle possède, mais comme une relation entre les personnes. Sur la base d'une telle explication, les mécanismes actuels de la distribution économique ne peuvent plus être considérés comme des mécanismes naturels, ni la plus grande richesse comme une récompense bien méritée d'un point de vue moral et les principes de la justice économique ont plus de chance d'inclure les principes plus étendus de la justice sociale.

Ce que montre le contraste entre ces deux explications, c'est d'abord que la première explication de la distribution de la richesse contient une présupposition structurale. En effet si, appliquant le test proposé par Garfinkel, nous nous demandons s'il est raisonnable de croire que dans les sociétés actuelles, toutes les personnes également talentueuses, également disposées à l'effort ou également capables de s'adapter à leur environnement jouissent d'une situation économique approximativement égale, la réponse évidente est : non. D'autres facteurs sont donc à l'oeuvre dans la distribution de la richesse, des facteurs qui sont présupposés par l'explication individualiste et qui sont constitutifs du phénomène que l'on cherche à expliquer. Ce sont ces facteurs qui sont pris en compte dans la deuxième explication. Alors que la 
première explication cherche à rendre compte de la distribution de la richesse en expliquant comment les individus en viennent à posséder ce qu'ils possèdent, la seconde en rend compte en expliquant comment, A et non pas B, en vient à occuper telle position au sein d'une distribution donnée de la richesse.

La seconde chose que montre le contraste entre ces explications est l'incidence qu'elles exercent sur le contenu et sur la portée des principes moraux qu'elles contribuent respectivement à justifier. D'une part, on peut remarquer que l'explication systémique, selon laquelle la richesse des uns dépend de la pauvreté des autres, rend le statu quo plus difficile à justifier d'un point de vue moral qu'il ne l'est sur la base de l'explication individualiste. Mais ce type de contrainte n'est ni le plus important ni le plus significatif. L'incidence la plus considérable, et aussi la plus implacable, qu'exerce l'explication sur les contenus moraux découle du fait qu'elle détermine la portée des principes de justice; en se portant garante de l'identification des facteurs de distribution elle désigne du même coup ce qui, dans une théorie donnée, doit faire l'objet d'une évaluation morale. Sur la base de la seule explication individualiste, il n'y a aucune raison de réfléchir moralement aux sources d'inégalité économique que pourrait révéler l'explication systémique comme, par exemple, la discrimination raciale ou sexuelle, l'exploitation ou encore l'existence d'un système de caste. Tenues hors de la portée de l'explication individualiste, précisément parce que celle-ci recèle des présuppositions structurales, ces sources d'inégalités sont aussi tenues à l'extérieur du domaine de la justice économique comme autant de facteurs non problématiques du point de vue de la justice. Le résultat est donc que ces sources d'inégalité économique sont cautionnées moralement, de même que le système qui les génère, sans pour autant avoir fait l'objet d'une évaluation morale en bonne et due forme. C'est là l'effet le plus percutant des explication recelant des présuppositions structurales lorsqu'elles interviennent dans la justification des jugements moraux.

2- Mon deuxième exemple des présuppositions structurales à l'oeuvre dans la théorie morale est celui d'un argument utilisé par Rawls à l'appui du principe de différence ${ }^{14}$. Il s'agit d'un argument qui fait un appel sans équivoque à deux axiomes de la théorie du choix social. Cette théorie formelle, développée dans le champ de l'économie de bien-être, a comme point de départ intuitif l'idée qu'un ensemble de politiques sociales (constituant une " option sociale " parmi d'autres) peut être justifié si on peut montrer qu'il serait choisi, de préférence à tout autre, par les agents rationnels susceptibles d'être touchés par ces politiques. Dans cette optique, le « choix social » s'explique par la convergence des choix individuels vers une option donnée et le but de la théorie est précisément de représenter formellement les conditions dans lesquelles une telle convergence pourrait être réalisée. Or la formulation axiomatique de ces conditions vient ajouter une coloration bien particulière à

14. Rawls, 1971 
l'idée intuitive du choix social. Le premier des deux axiomes qui m'intéressent ici, stipule l'indépendance des préférences individuelles : les préférences qu'entretient $A$ ne dépendent pas du fait que $B$ entretienne les préférences qu'il entretient et conversement. Un second axiome stipule l'indépendance des biens, c'est-à-dire de ce qui est préféré par les agents : le x préféré par $\mathrm{A}$ n'a aucun effet sur l'utilité, pour B, du y qu'il préfère et conversement.

Ces deux axiomes ont des implications qu'il importe de souligner. Le premier axiome impliquerait, par exemple, qu'une option sociale sera choisie par un agent sans que celui-ci fasse entrer dans ses considérations la probabilité que cette option soit aussi voulue par d'autres que lui ; ainsi, les choix des agents doivent demeurer indépendants de toute croyance concernant les mérites et les démérites, pour l'ensemble du corps social, de l'option qu'ils préfèrent. Le second axiome, de son côté, signifierait par exemple, qu'une société où les travailleurs peuvent se donner des organisations syndicales efficaces, si telle est l'option favorite de A, devrait être vue comme ne posant aucune limite aux avantages que B pense pouvoir retirer d'une société régie par les lois d'une économie capitaliste et conversement ; ainsi, les choix des agents devraient demeurer entièrement indépendant de toute considération concernant la manière dont la vie en société peut modifier, ou contrecarrer leurs attentes. Bref, ces deux axiomes font en sorte que les seuls choix individuels capables de contribuer au choix social, soient ceux qui sont basés sur des préférences individuelles, elles-mêmes uniquement fondées sur des considérations individualistes et jamais sur des considérations ou des croyances portant sur autrui, ses intérêts ou sur les conflits qu'ils sont susceptibles de susciter au sein d'une société. L'explication du choix social, donnée dans ce contexte, véhicule donc, par la voie de ces deux axiomes, son lot de présuppositions structurales. Les contraintes imposées par ces deux axiomes sont en fait des contraintes sur l'expression des préférences individuelles en matières sociales; elles font en sorte que les préférences susceptibles d'être motivées par des considérations systémiques ne peuvent tout simplement pas être prises en considération.

En vertu du principe de différence de Rawls, les inégalités sont moralement acceptables au sein d'une société juste si elles contribuent à l'amélioration de la condition des moins bien nantis. Pour montrer que ce principe est justifié, Rawls suppose deux options sociales et argue que les choix d'individus rationnels convergeraient vers la première. Dans cette première option la distribution du bien-être s'effectue, disons, selon une échelle qui va de 6 pour les mieux nantis à 5 pour le groupe médian puis à 4 pour les moins bien nantis. La deuxième option illustre une société strictement égalitariste ou le niveau de bien-être se situe à 3 . Rawls soutient que le choix d'individus rationnels convergent vers la première option car celle-ci représente, par rapport à la deuxième, une amélioration du sort de chacun, incluant celui des moins bien nantis. Ce ne sont pas des considérations portant sur les avantages individuels des agents, mais l'envie seule, dit-il, qui pourrait motiver le 
rejet de la première option ; mais puisque l'envie, ajoute Rawls, n'est pas un motif valable d'un point de vue moral, alors la première option doit prévaloir ${ }^{15}$.

Le raisonnement de Rawls, pourrait donc être reconstruit de la façon suivante : i) chaque individu jouit d'un plus grand bien-être dans la première option que dans la seconde. ii) chaque individu a donc des raisons personnelles, qui ne doivent rien aux préférences d'autrui, de choisir la première option. Ainsi, l'indépendance des préférences prévaut. iii) pour deux individus $\mathrm{A}$ et $\mathrm{B}$ ayant des niveaux de bien-être différents dans la première option, l'amélioration de la condition de $\mathrm{A}$ par rapport à la deuxième option, n'a aucun effet sur l'amélioration escomptée par B. Ainsi formulée, l'idée de Rawls selon laquelle la première option n'est préjudiciable pour personne ( "seule l'envie... ») apparaît comme un corollaire de l'axiome stipulant l'indépendance des biens. Rawls semble donc prendre pour acquis que ce raisonnement est fondé puisqu'il satisfait aux deux conditions posées les axiomes dont il a été question plus tôt.

Mais n'eût été de ces conditions, le scénario décrit par Rawls aurait pu se prêter à un raisonnement bien différent, accréditant le choix de l'option égalitariste et justifiant par le fait même le principe de justice correspondant. Des individus rationnels, non limités par la condition découlant de l'indépendance des biens, auraient pu réaliser que certains tirent un plus grand avantage que d'autres de la première option ${ }^{16}$. Réfléchissant au fait que cette option ne peut pas satisfaire les attentes maximales de tous, et en particulier leurs propres attentes, et craignant peut-être les conflits sociaux qui pourraient en résulter, ils auraient pu choisir la seconde option. Et des individus rationnels, non limités par la condition résultant de l'indépendance des préférences auraient pu constater qu'une distribution inégalitariste avait pour effet l'attribution à différents individus d'un rang différent. Ils auraient pu faire intervenir dans leur raisonnement les avantages et les inconvénients marginaux résultant de la position relative qui échoit à chacun en vertu de cette distribution ${ }^{17}$, comme les inégalités de pouvoir ou la perte pour certains

15. Ibid., p. 531-536

16. En vertu de l'axiome stipulant l'indépendance des biens, on ne peut pas prendre en considération le fait que l'utilité d'un individu qui choisit la première option parce qu'il espère accéder au niveau supérieur dans cette option, soit affectée par le fait qu'un autre individu y accède à sa place. Mais puisque ceci doit être pris en considération lorsque l'on compare entre elles les utilités dévolues à chaque niveau dans la première option alors le seul raisonnement possible doit être basé, comme l'argument de Rawls, sur la présence/absence d'amélioration par rapport à la deuxième option et ceci n'implique qu'une comparaison entre le niveau d'utilité dans la deuxième option et les niveaux d'utilité, pris un à un, dans la première option.

17. A. K. Sen a souligné que l'argument de Rawls ne fait intervenir que des considérations de niveau. Il établit un contraste, sur ce point, entre le contractualisme et l'utilitarisme qui, lui, se base sur une comparaison des gains encourus par les uns et des pertes encourues par les autres. Sen soutient que les deux points de vue sont complémentaires et qu'il est essentiel de combiner les deux lorsque l'on aborde les questions de justice distributive. (Sen, 
de l'estime de soi et, au vue des risques encourus, opter pour une distribution égalitariste et ce, sans que l'envie n'entre en ligne de compte.

Plusieurs aspects de la théorie de Rawls nous invitent d'ailleurs à réfléchir en ces termes lorsqu'il est question d'inégalité socio-économique. Mais lorsque Rawls cherche à justifier le principe de difference, la question centrale, pour lui, est celle de savoir si chacun peut escompter un niveau de bienêtre plus élevé dans une société inégalitaire. La question qu'il aurait du poser dans ce contexte est plutôt celle de savoir si chacun a des raisons de penser qu'une distribution inégalitaire lui sera profitable. Mais cette seconde question n'est même pas formulable dans le modèle du choix rationnel que retient Rawls, car elle impliquerait la possibilité, d'emblée exclue par ce modèle, d'une incursion dans le domaine des effets systémiques de la distribution. Si Rawls en arrive à la conclusion - étrange, compte tenu de ce qu'il dit par ailleurs des effets de l'inégalité sociale - que l'inégalité socio-économique peut être moralement justifiée dans certaines circonstance, c'est parce qu'il fait appel à une explication du choix rationnel qui détermine le cadre à l'intérieur duquel les agents doivent faire des choix, mais qui ne cherche pas à expliquer comment ou pourquoi des agents rationnels choisiraient de délibérer à l'intérieur d'un tel cadre. Si l'on soulevait sérieusement la question de savoir quels motifs pourraient avoir des agents rationnels pour restreindre ainsi le cadre de leurs délibérations, la réponse serait sans doute : aucun. Ce qui montrerait bien, s'il en était encore besoin, que ce modèle de la rationalité recèle des présuppositions structurales. Mais la justification morale du principe de différence hérite de ces présuppositions. Comme le modèle de la rationalité utilisé par Rawls ne permet pas de rendre compte de certains types de considérations dont on peut penser qu'elles font partie du raisonnement d'agents rationnels, ces considérations sont aussi exclues de celles qui devraient entrer dans l'évaluation du principe de différence. Mais ce qui est exclu de cette manière n'est pas pour autant laissé pour compte. Bien au contraire, les effets systémiques de la distribution, sur lesquels le modèle de la rationalité de Rawls nous force à fermer les yeux, sont par le fait même tenues pour inoffensifs du point de vue de la justice et donc, moralement cautionnés.

\section{Les limites de l'enquête philosophique}

Le problème soulevé en théorie morale par les explications qui recèlent des présuppositions structurales est donc double. D'abord ces explications mettent l'accent sur certains aspects du phénomène à expliquer tout en négligeant certains autres aspects de ce phénomène. Elles induisent de ce fait une perspective

non daté). Mon propre argument souligne la pertinence, dans ce contexte, d'un troisième point de vue : celui où on peut comparer les niveaux en tenant compte de leur valeur relative au sein d'une distribution. Je remercie Nicolas Kaufmann pour avoir attiré mon attention sur cet article de Sen qui m’a permis de formuler plus précisément la position que j'avance ici. 
particulière qui délimite le domaine explicite de la justification morale. Mais ce faisant, et c'est la deuxième facette du problème, elles excluent de ce domaine les aspects négligés du phénomène qu'elles expliquent, avec comme résultat que ceux-ci sont, en dernière analyse, moralement cautionnés sans pour autant faire l'objet d'une justification morale en bonne et due forme.

La question que je voudrais soulever maintenant, et ceci nous ramène sur le terrain d'une théorie de la justification, est la suivante : Si, comme je l'ai soutenu dans la première section de cet article, la justification des jugements moraux particuliers peut être validée aux termes d'une conception donnée de la justification, peut-on s'attendre à ce que les explications recelant des présuppositions structurales et contribuant à la justification des jugements moraux particuliers, soient accréditées par certaines conceptions de la justification ? Supposons, par exemple, qu'une conception de la justification élaborée au terme de l'enquête philosophique ait comme conséquence que les seules explications pouvant contribuer à la justification morale proprement dite sont en fait des explications recelant des présuppositions structurales ; quelle conclusion pourrait-on en tirer, et au sujet de cette théorie de la justification, et au sujet de la validité des explications qu'elle requiert ? L'importance de répondre à cette question apparaît clairement lorsque l'on réfléchit au fait que les théories de la justification, du moins comme je les ai caractérisées, s'appuient en grande partie sur des explications de la moralité. Si l'on en venait à conclure qu'est inacceptable une conception de la justification qui requiert, au niveau de la justification des jugements moraux particuliers, des explications recelant des présuppositions structurales, cela pourrait bien signifier que certaines explications de la moralité ne sont pas non plus acceptables.

La réponse à cette question peut sembler évidente, mais je crois qu'elle ne l'est qu'en partie. Si une théorie donnée de la justification morale requiert que les contenus moraux soient en partie soustraits à la justification proprement dite, il est en effet évident qu'elle est inacceptable. Mais ceci, comme je l'ai souligné plus tôt, n'est qu'un aspect du problème soulevé, en théorie morale, par les explications contenant des présuppositions structurales. La réponse à la question devient moins évidente lorsque cette question porte sur la perspective particulière induite par une explication contribuant à la justification morale. Que pourrait-on conclure, pas exemple, d'une théorie de la justification exigeant que les seules explications valides susceptibles de contribuer à la justification de jugements moraux particuliers soient des explications individualistes ? Ces explications, comme je l'ai déjà dit, contiennent la plupart du temps des présuppositions structurales. Devrions-nous conclure que leur usage dans le cours de la justification morale peut être validé par une théorie de la justification ou devrait-on plutôt conclure que la théorie est elle-même inacceptable?

A quoi pourrait ressembler, pour commencer, une telle théorie de la justification ? Elle devrait d'abord articuler, sur la base d'une explication de 
la moralité ou de certains de ces aspects, une analyse qui établit la primauté d'un point de vue individualiste et justifier cette analyse en regard des explications rivales, c'est-à-dire des explications en vertu desquelles, par exemple, les notions de collectivité ou de contexte social doivent jouer un rôle de premier plan. Ce faisant une explication individualiste de la moralité souscrit aux standards de l'enquête philosophique c'est-à-dire, qu'elle cherche à s'appuyer sur des arguments plausible et valides, et prend en considération les objections possibles. Mais elle élucide du même coup la perspective qui, selon elle, doit prévaloir dans une justification des jugements moraux particuliers et elle le fait non seulement en articulant et en défendant cette perspective, mais en rendant explicite ce qu'elle exclut, c'est-à-dire, ce qu'elle estime être de mauvaises raisons à l'appui des jugements moraux particuliers. C'est dire qu'au niveau où s'articule une théorie de la justification, les présuppositions structurales des explications requises par cette théorie sont entièrement explicitées et que cette théorie n'en fait elle-même intervenir aucune, au niveau de son explication de la moralité, qui n'ait d'abord été explicitement motivée.

Jusqu'ici, une théorie de la justification ainsi conçue pose donc des contraintes sur la nature des raisons susceptibles de contribuer à la justification des jugements moraux particuliers, mais elle n'en pose aucune sur le domaine des objets susceptibles d'être soumis à une évaluation morale. Si cette théorie stipule que seules des explications individualistes peuvent légitimement intervenir dans la justification morale, elle ne requiert pas qu'aucun argument (individualiste) soit déployé, pour montrer, par exemple, la non-pertinence morale de facteurs systémiques. C'est-à-dire qu'elle ne requiert pas que les facteurs exclus du domaine des « bonnes raisons " par la perspective individualiste, demeurent aussi à l'abri d'une évaluation morale.

Mais ce qui n'est pas exigé par une théorie de la justification est-il permis par elle ? La justification morale pourrait-elle être validée par le simple fait qu'elle est compatible avec une théorie individualiste de la justification et, sous prétexte que cette dernière rend explicite la perspective individualiste et ce qu'elle exclut, être dispensée de soumettre à une évaluation proprement morale des jugements que par ailleurs elle cautionne moralement ? Autrement dit, la justification morale peut-elle prendre prétexte d'une conception de la justification pour faire usage d'explications contenant des présuppositions structurales? Prétendre que oui serait prétendre à l'équivalence de deux ordres différents de normativité : celui où l'on détermine ce qui doit compter pour une bonne raison et celui où l'on donne des raisons particulières à l'appui de jugements moraux particuliers. Si la justification morale mise ainsi sur les présupposés structuraux d'une explication individualiste, ce ne peut être qu'à la faveur d'une confusion entre ces deux ordres de justification, mais une fois dissipée la confusion, il devient clair que ce que la théorie de la justification n'exige pas, ne peut pas non plus être validé en son nom. Et ce qu'elle n'exige pas ne peut pas non plus réussir à l'invalider. Il semble 
donc qu'une conception philosophique de la justification, qu'elle soit basée ou non sur une explication individualiste, ne peut pas, si elle respecte les standards de l'enquête philosophique, exiger de la justification des jugements moraux particuliers qu'elle fasse appel à des explications contenant des présuppositions structurales.

Nous pouvons donc conclure que des conceptions de la justification qui exigeraient de la justification morale qu'elle fasse appel à des explications recelant des présuppositions structurales sont inacceptables. De telles conceptions, je l'ai déjà dit, sont inacceptables en regard de leurs conséquences parce qu'elles impliquent que les contenus moraux soient en partie soustraits à la justification morale proprement dite. Mais, de plus, il est difficile d'imaginer comment elles pourraient avoir une telle conséquence sinon en se basant ellesmêmes sur des explications de la moralité qui font intervenir des présupposés moraux ou structuraux. Comme nous venons de le voir, c'est un écueil que doivent réussir à éviter les conceptions crédibles de la justification morale.

C'est dire que dans aucun cas l'usage d'explications contenant des présuppositions structurales ne peut être accrédité aux termes d'une enquête philosophique : ou bien celle-ci est menée conformément aux standards philosophiques appropriés, et alors elle ne peut pas servir de caution à de telles explications, ou bien elle n'est pas conduite conformément à de tels standards et alors elle ne peut rien cautionner du tout.

Ce résultat est encourageant, car ce qu'il tend à confirmer, s'il est bien fondé, c'est que la critique que j'ai faite ici des explications recelant des présuppositions structurales et du rôle qu'elles jouent dans la justification des jugements moraux particuliers ne présume en rien de la valeur des conceptions substantives concernant soit la nature de la moralité soit le contenu de la morale. Cette critique se fonde entièrement sur une caractérisation générale de ce qu'est la justification morale, et mes conclusions, sur le rapport que celle-ci entretient, au sein de la philosophie morale, avec les explications de la moralité.

\section{Conclusion}

S'il était important d'établir le bien fondé de cette analyse en regard de l'économie générale de la théorie morale, il faut cependant souligner que l'usage d'explications recelant des présuppositions structurales dans le cours de la justification de jugements moraux particuliers, ne se revendique habituellement pas d'une conception philosophique de la justification. Comme dans le second exemple que j'ai présenté ici, l'usage de ce type d'explication est plutôt vu comme un moyen commode de représenter certains types de raisonnement et d'étayer, du même coup, des jugements moraux particuliers. Mais dans ce contexte, ces explications risquent d'autant plus d'avoir des effets pervers; non seulement elles ont un impact sur les contenus moraux de la théorie, mais encore, cet impact risque de passer inaperçu aux yeux de ceux-là mêmes qui font usage de telles explications, tenant dès lors pour justifié ce qui en fait ne 
l'est pas et ne pourrait pas l'être si l'on s'en remettait aux conceptions explicites de la justification et aux prémisses morales explicitement acceptées par ces auteurs. Il est remarquable que Rawls, dont on a l'habitude de dire en exagérant un peu que sa théorie de la justice est en fait une théorie de la justification morale, s'en soit remis, pour ce qui est de la justification de ses deux principes de justice, à un mode de représentation du raisonnement moral dont les présupposés structuraux, à supposer qu'ils aient jamais été clairement identifiés par lui, n’auraient jamais passé le cap de sa monumentale conception de la justification, ni contribué à justifier un principe de différence qu'aucune des prémisses morales admise par Rawls ne pouvait impliquer.

Les présuppositions structurales ne sont probablement qu'un indice parmi d'autres de l'impact qu'exerce subrepticement l'appareil méthodologique des théories morales sur les contenus moraux. Cela reste à explorer. Tout ce que j'ai voulu montrer ici, c'est qu'il y a au moins quelques bonnes raisons, pour les philosophes moraux qui sont de plus en plus enclins aux emprunts méthodologiques, d'entreprendre une telle exploration. Ces raisons tiennent à la clarification de ce que l'on entend, et de ce que l'on peut entendre, en théorie morale, lorsque dit qu'un principe ou qu'un jugement moral est justifié. Une raison supplémentaire de le faire, qu'il n'y avait pas lieu d'élaborer dans le contexte du présent article mais à laquelle les philosophes devraient être sensibles, est illustrée par le cas des explications recelant des présuppositions structurales. Ces explications sont, par définition, des explications inadéquates du point de vue empirique. Compte tenu des contraintes qu'elles font peser sur les contenus moraux proprement dits, on peut difficilement s'attendre à ce que les jugements moraux justifiés sur la base de telles explications soient compatibles, comme le souhaitent plusieurs philosophes, avec nos meilleures représentations du monde dans lequel nous vivons. Que l'on soit ou non compatibiliste, cet exemple montre que nous avons aussi intérêt à réfléchir au fait que ce que l'on considère comme de simples emprunts méthodologiques peuvent faire d'une entreprise de justification morale, un exercice d'autoréfutation.

\section{Bibliographie}

Daniels, Norman, 1996, Justice and Justification, Cambridge, Cambridge University Press.

Dretske, Fred, 1973, "Constrastive Statements ", Philosophical Review 82.

Garfinkel, Alan, 1981, Forms of Explanation, New Haven, Yale University Press.

Gauthier, David, 1986, Morals by Agreement, Oxford, Clarendon Press.

Mackie, J. L., 1977, Ethics : Inventing Right and Wrong, Harmondsworth : Penguin.

Nielsen, Kai, 1996, Naturalism without Foundations, Amherst, Prometheus Books. 
Rawls, John, 1971, A Theory of Justice, Harvard, Harvard University Press. Scanlon, Thomas, 1992, "The Aims and Authority of Moral Theory ", Oxford Journal of Legal Studies, Vol. 12, no.1. 1-23

Sen, A. K., (non daté), "Rawls versus Bentham: An Axiomatic Examination of the Pure Distribution Problem » dans Daniels, Norman dir., Reading Rawls, New York, Basic Book. 